\title{
In vivo human retinal and choroidal vasculature visualization using differential phase contrast swept source optical coherence tomography at $1060 \mathrm{~nm}$
}

\author{
Reza Motaghiannezam and Scott Fraser \\ Beckman Institute, Division of Biology, California Institute of Technology, Pasadena, \\ California 91125-7400
}

\begin{abstract}
A differential phase contrast (DPC) method is validated for in vivo human retinal and choroidal vasculature visualization using high-speed swept-source optical coherence tomography (SS-OCT) at $1060 \mathrm{~nm}$. The vasculature was identified as regions of motion by creating differential phase variance (DPV) tomograms: multiple B-scans were collected of individual slices through the retina and the variance of the phase differences was calculated. DPV captured the small vessels and the meshwork of capillaries associated with the inner retina in en face images over $4 \mathrm{~mm}^{2}$ in a normal subject. En face DPV images were capable of capturing the microvasculature and regions of motion through the inner retina and choroid.
\end{abstract}

Keywords: Optical coherence tomography, Fourier Domain, Coherence imaging, Medical and biological imaging, Optical Coherence Angiography, Ophthalmology

\section{INTRODUCTION}

The importance of retinal and choroidal vasculature visualization is paramount in diagnosing various eye diseases, such as diabetic retinopathy ${ }^{1}$, age-related macular degeneration (AMD) ${ }^{2}$, glaucoma ${ }^{3}$, and anterior ischemic optic neuropathy $(\mathrm{AION})^{4}$.

Color fundus photography (CF) and fluorescein angiography (FA) have served as the gold standard methods for imaging the retinal vasculature network ${ }^{5}$. Indocyanine green angiography (ICGA) extends such imaging to the deeper choroidal vessels ${ }^{5}$. However, these imaging techniques are limited by the 2-D nature of the images they collect (lacking depth information) and/or their inability to capture the deep choroidal blood vessels.

To meet the need for 3D retinal and choroidal vasculature assessment without the use of fluorescent dye injection, optical coherence tomography (OCT) has emerged as an attractive non-invasive depth-resolved imaging technology ${ }^{6}$. OCT realizes 3D visualization of retinal structure ${ }^{7}$ and vasculature ${ }^{8}$ by capturing interferograms of the light reflected back from the retina. Doppler OCT (D-OCT) ${ }^{9}$ and Phase Contrast (PC)-OCT ${ }^{10}$ are motion sensitive methods for vessel visualization, since these methods collect more than one depth scan of the same retinal loci and analyze Doppler phase shifts between depth scans at different depths. Although D-OCT captures the regions of high-velocity blood flow, such as in major vessels, it is unable to capture slow flow in retinal capillaries ${ }^{9}$ or deep flows such as the choroidal circulation due to limited phase sensitivity and small time separation between two successive A-scans.

Smart scanning protocols enhance sensitivity to the smaller signals expected from the microvasculature by increasing the time separation between two OCT depth scans and relying on the acquired phase ${ }^{10}$ or joint intensity and phase information ${ }^{11,12}$ of $800 \mathrm{~nm}$ spectral domain (SD)-OCT signals for contrast. The proposed motion contrast method by Wang $^{11}$ can visualize the microvasculature using Hilbert and Fourier analyses of the OCT signal; however, these also highlight highly reflective stationary regions. 2D Fourier analysis of the OCT signals along the wavenumber and time axes can provide structural and motion information for mapping the blood flow velocity within retinal capillaries ${ }^{12}$. Our previous work captured the retinal microvasculature by collecting several phase change tomograms and finding region of motion from variance analysis ${ }^{10}$. However, in each of these tools, the inner choroid was not captured well using phase contrast SD-OCT at $800 \mathrm{~nm}$ due to its shallow penetration depth and sensitivity roll-off.

There is an important need for OCT systems with the required imaging depth and phase sensitivity for capturing retinal and choroidal microvasculature. To enhance imaging as deep as the choroid, $1 \mu \mathrm{m}$ Fourier domain OCT has been

Optical Coherence Tomography and Coherence Domain Optical Methods in Biomedicine XVI,

edited by Joseph A. Izatt, James G. Fujimoto, Valery V. Tuchin, Proc. of SPIE Vol. 8213, 821304

(C) 2012 SPIE · CCC code: $1605-7422 / 12 / \$ 18 \cdot$ doi: $10.1117 / 12.910991$

Proc. of SPIE Vol. $8213821304-1$ 
suggested as a preferred technology ${ }^{13}$. Images collected by SD-OCT at $1 \mu \mathrm{m}$ can delineate the multiple choroidal layers and capture the choroidal vessels distribution, size, density, and blood perfusion ${ }^{14}$. Retinal microvasculature has also been imaged with an elegant dual-beam SD-OCT system, capturing the effective frequency shift between two detected OCT signals at $1020 \mathrm{~nm}^{15}$. However, the system's complexity, cost, and 3-dB sensitivity loss may limit its clinical application.

Superior detection efficiency and sensitivity roll-off performance of swept source (SS)-OCT makes this Fourier domain method more attractive than SD-OCT for choroidal visualization. In fact, a SS-OCT at $1.3 \mu \mathrm{m}$ shows the power of the phase contrast method to capture deep vasculature in the brain and tumors ${ }^{16}$; however, the significant absorbance of 1.3$\mu \mathrm{m}$ light by the vitreous limits the use of this approach for retinal and choroidal vasculature imaging. To combine the superior penetration depth and depth sensitivity, we have developed a SS-OCT $1060 \mathrm{~nm}$ system with the needed phase sensitivity and scan speed to capture phase variance data from the inner retina and choroid.

Motion contrast was achieved in this paper, as in our previous work ${ }^{10}$, by measuring the phase difference between successive B-scans. We removed one of the inherent phase error sources in SS-OCT, by eliminating timing-induced phase error ${ }^{17}$. Differential phase variance (DPV) ${ }^{10}$ method was implemented for assessing the retinal microvasculature and regions of motion in the inner choroid. DPV was able to capture the foveal avascular zone (FAZ) and image the choroidal vasculature.

\section{METHODS}

\subsection{SS-OCT system}

To validate the DPV method for providing motion contrast, we used a prototype $50 \mathrm{kHz}$ phase sensitive SS-OCT system, incorporating a polygon-based $1060 \mathrm{~nm}(1015-1103)$ swept laser source ${ }^{18}$, with $\sim 5.9 \mu \mathrm{m}$ axial resolution in tissue and $102 \mathrm{~dB}$ sensitivity (1.2 $\mathrm{mW}$ incident power). The SS-OCT system was comprised of the polygon-based swept-laser source $^{18}$, an interferometer, and a data acquisition (DAQ) unit (Fig. 1). The swept source output was coupled to the interferometer through an isolator where a 90/10 coupler was used to split light into a sample arm : reference arm. The sample arm light was split equally between the calibration arm and a slit lamp biomicroscope (Carl Zeiss Meditec) as shown in Fig. 1. A 50/50 coupler combined and directed the reflected light from the sample to the one port of the interferometer output coupler. The reference arm light passed through a pair of collimators and was directed to the second port of the interferometer output coupler. The resulting interference fringes were detected on both output ports using a dual balanced photodetector. To generate a trigger signal at the beginning of the first interference fringe for data acquisition, a portion of the reference arm light was directed to a three port circulator and a fiber Bragg grating. A reflected optical pulse was detected using a photodetector and converted into an electronically tunable TTL signal as a trigger signal. The spectral signals were continuously digitized by triggering an $\mathrm{AD}$ conversion board. A D/A board was used to generate the driving signals of the two-axis galvanometers. A user interface and data acquisition was developed in LabView to coordinate instrument control and enable user interaction.

\subsection{Scanning protocols}

The prototype SS-OCT instrument was used to image the eye of a healthy volunteer (37-year-old Caucasian male) at the California Institute of Technology (Caltech). This study was approved by the Caltech committee for the protection of human subjects and adhered to the tenets of the Declaration of Helsinki. Written informed consent was obtained from subjects prior to OCT imaging. Total exposure time and incident exposure level were kept less than 5.5 seconds and 1.2 $\mathrm{mW}$ in each imaging session, consistent with the safe exposure determined by American National Standards Institute (ANSI) and International Commission on Non-Ionizing Radiation Protection (ICNIRP). Patient interfaces were based on a Stratus OCT-3 system (Carl Zeiss Meditec) adapted with optics to support the $1060 \mathrm{~nm}$ wavelength range. A 60-D lens was used at the exit of the fundus camera with $13 \mathrm{~mm}$ working distance providing a beam diameter of $1.5 \mathrm{~mm}$ on the cornea ( $\sim 15 \mu \mathrm{m}$ transverse resolution).

Two scanning protocols were implemented. A 2D protocol acquired four horizontal tomograms (B-scans) with 201 depth scans (A-scans) spanning the same transverse slice $(2 \mathrm{~mm})$ across the foveal centralis in 0.02 seconds. In the second protocol, a 3D OCT data set was collected by acquiring several neighboring B-scans over the parafovea. The system magnification, SS-OCT speed $(50400 \mathrm{~Hz})$, speed of the fast scan axis $(200 \mathrm{~Hz})$ with fly-back time $(1 \mathrm{~ms})$, and data acquisition time (4 seconds) gave an image size of $201 \times 200$ pixels over a $2 \mathrm{~mm} \times 2 \mathrm{~mm}$ field of view (FOV); each Bscan was repeated four times. In the 3D scanning protocol, the fast scan axis was sagittal (superior-inferior) and the slow axis was horizontal (nasal-temporal). 


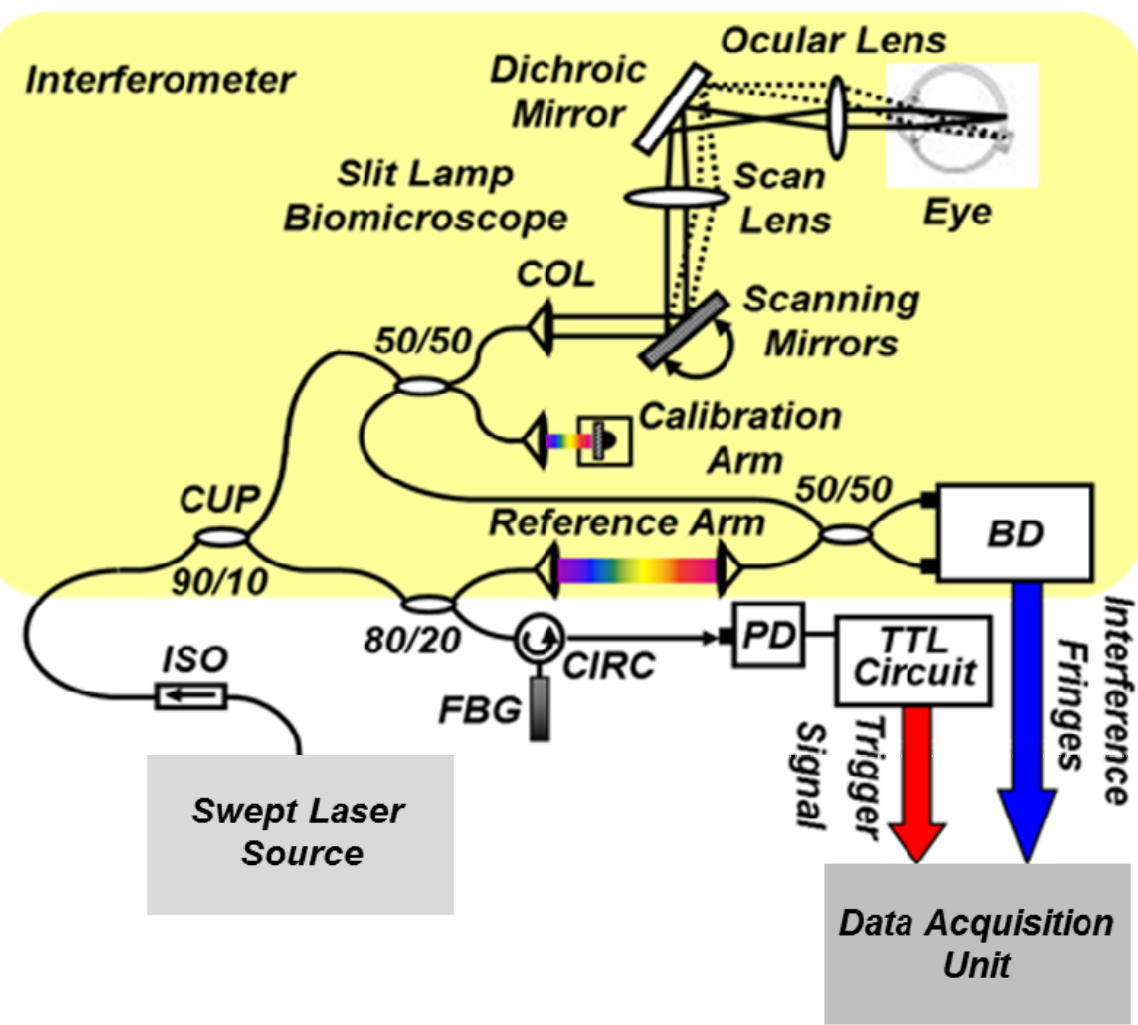

Fig. 1. Schematic of a polygon-based swept-laser source (gray box), interferometer (yellow box), and SS-OCT data acquisition unit (dark gray box) used for imaging. COL, collimator; PC, polarization controller; ISO, isolator; CIRC, circulator; CUP, coupler; PD, photodetector; BD, dual balanced photodetector ( $\mathrm{InGaAs}, 80 \mathrm{MHz})$; FBG, fiber Bragg grating $(0.1 \mathrm{~nm})$. Data acquisition unit comprising of an AD conversion board (14-bit, GaGe CompuScope 14,200) for digitizing interference fringes and a

D/A board (16 bit, National Instruments) for driving scanning mirrors (Cambridge Technology).

\subsection{Image processing and DPV imaging}

The digitized signals were divided into individual spectral sweeps in the post-processing algorithm. Equal sample spacing in wave number $(k)$ was achieved using a calibration trace at $1.5 \mathrm{~mm}$ interferometer delay and numerical correction of the nonlinearly swept waveforms ${ }^{20}$. Image background subtraction and numeric compensation for second order dispersion ${ }^{21}$ were performed. The complex SS-OCT data sets were upsampled by a factor of 4 and Fourier transformed. Axial motion correction was achieved on the obtained 2D and 3D SS-OCT data sets by cross correlating the consecutive horizontal tomograms.

To perform motion contrast analysis and imaging, multiple B-scans were acquired over the same slice. Time separations were $T_{B}=5 \mathrm{~ms}$ between B-scans for capturing the same position, respectively. Multiple phase measurements were recorded over the same transverse point separated in time. DPV captured the differences between multiple phase measurements $(\varphi(z, T))$ of the same transverse points (separated in time) and calculated the variance of these changes (Fig. 2). To measure and remove timing-induced phase error ${ }^{17}$ due to the random delay between the trigger signal and the subsequent A-to-D conversion (sample clock), a calibration signal was generated using a stationary mirror in the calibration arm (Fig. 1) as described by Vakoc ${ }^{17}$. The calibration signal was located at a depth of $2 \mathrm{~mm}$ in the OCT intensity image. The corrected phase differences between adjacent B-scans for the same transverse point at a given depth were calculated by subtracting the phase difference of the calibration signal, linearly scaled with the sample signal depth, from the measured phase differences ${ }^{17}$ (Fig. 2). Phase unwrapping was performed on all measurements. A weighted mean algorithm ${ }^{19}$ estimated and removed the bulk axial motion phase change error (Fig. 2).

The same described procedures were repeated for the adjacent transverse points in the same and neighboring B-scans to capture the retinal vasculature in $2 \mathrm{D}$ and $3 \mathrm{D}$ data sets. To remove SNR-limited intensity and phase change errors in 2D 
and 3D data sets for vasculature visualization, an average intensity threshold ( $10 \mathrm{~dB}$ above the mean value of the noise floor) was applied; all contrasts with average intensity values $<$ mean $\left(\log _{10}\left(\mathrm{I}_{\text {noise }}\right)\right)+10 \mathrm{~dB}$ were set to zero in the corresponding images (Fig. 2).

To create the retinal and choroidal en face views, the inner/outer photoreceptor segments (IS/OS) and retina pigment epithelium (RPE) as well as vitreoretinal interface were detected using a segmentation algorithm ${ }^{8}$. Two depth integrated motion contrast en face images were generated by integrating the DPVs in the inner retina and in the inner choroid.

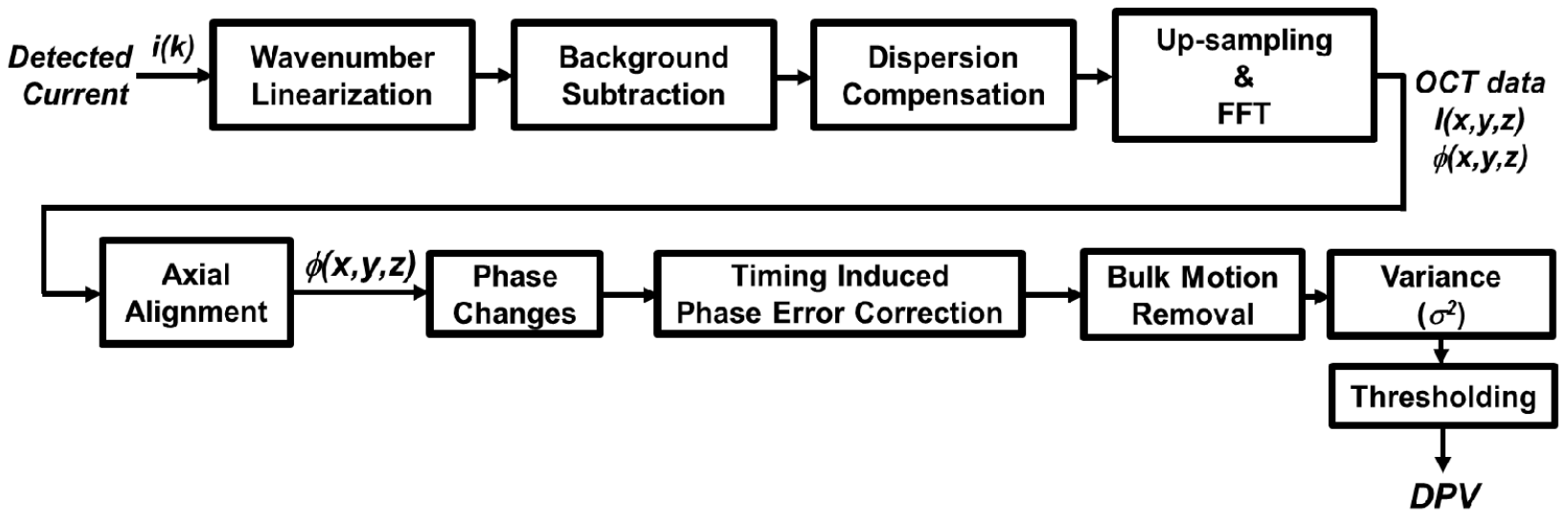

Fig. 2. Flowchart representing the data processing procedures for generating DPV.

\section{RESULTS}

\subsection{D tomogram visualization of the retina/choroid using DPV imaging method}

To study DPV method, four B-scans were acquired across the foveal centralis $(2 \mathrm{~mm})$. The averaged intensity of four obtained B-scans is depicted in Fig. 3(a). 2D DPV tomograms are shown in Figs. 3(b)-3(c) before and after compensation, respectively. Figure 3(b) demonstrates DPV is unable to capture motion without use of compensation algorithms ${ }^{17,19}$ and an extra hardware module ${ }^{17}$. Regions of motion in the inner choroid (white band) and the small vessel in the inner retina (white arrow) are detectable in this 2D tomogram (Fig. 3(c)).
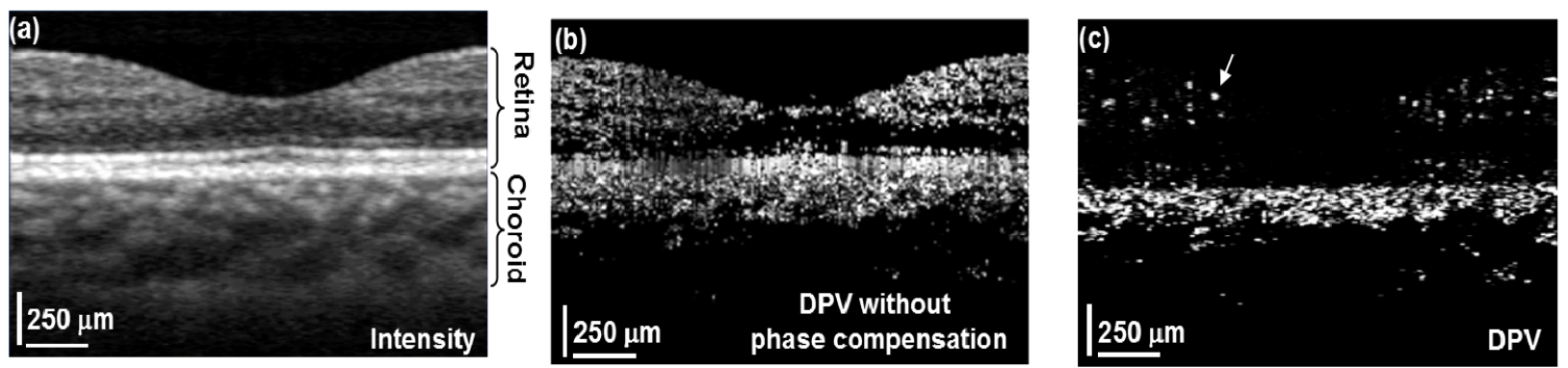

Fig. 3. Foveal (a) average intensity, (b) DPV before phase compensation, and (c) DPV after phase compensation 2D tomograms (2 $\mathrm{mm})$. White regions correspond to regions with higher either motion or/and reflectivity. White arrow indicates the small vessel in Fig. $3(\mathrm{c})$.

\subsection{En face view visualization of the retina and choroid using DPV method}

Figures 4(a)-4(b) illustrate the intensity and DPV en face views generated by integrating their values between the region $30 \mu \mathrm{m}$ posterior to the vitreoretinal interface and the region $130 \mu \mathrm{m}$ anterior to IS/OS. Figure 4(a) shows that the meshwork of capillaries is barely visible in the intensity en face view. Motion contrast enhancement is depicted in Fig. 4(b) using DPV method. Blood vessels in the ganglion cell layer and capillary meshwork of the inner plexiform layer are visualized in the DPV en face view (Fig. 4(b)). FAZ is resolvable by considering the capillary network around it as shown in the DPV image in Fig. 4(b). 


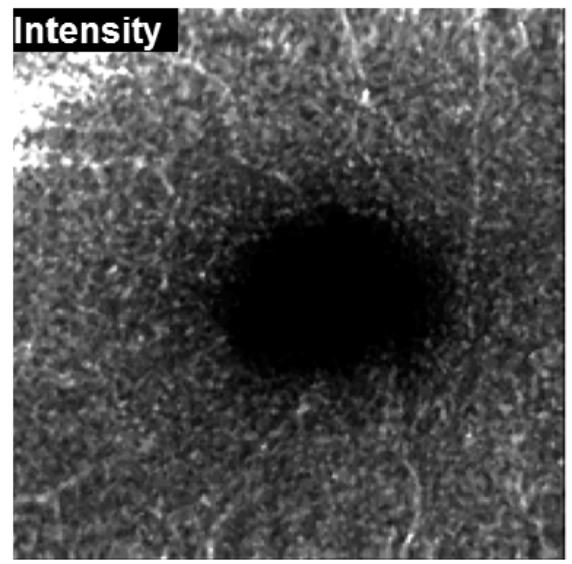

(a)

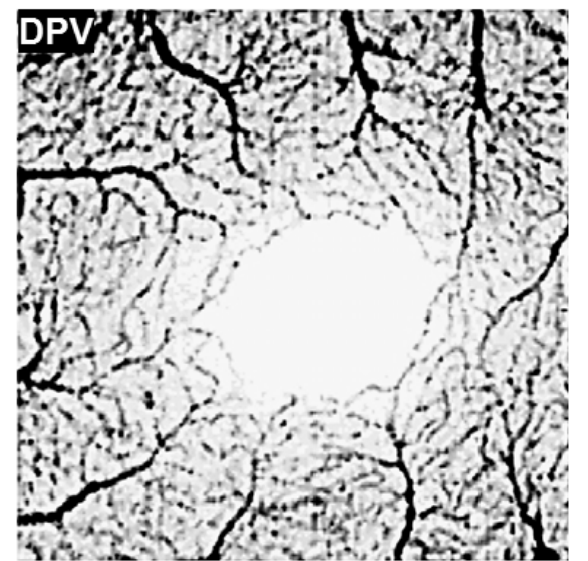

(b)

Fig. 4. Parafoveal depth-integrated en face views over $4 \mathrm{~mm}^{2}$ FOV acquired in 4 seconds. (a) Averaged intensity and (b) DPV en face images of the inner retina.

To depict structure and regions of motion in the inner choroid, the depth-integrated intensity and DPV en face images were generated by summing intensities and DPVs over $25 \mu \mathrm{m}$ thickness located $25 \mu \mathrm{m}$ beneath Bruch's membrane contour in the Sattler's layer (SL) (Figs. 5(a-b)). SL structure and regions of motion are visualized in the linear intensity (Figs. 5(a,c)) and inverted DPV (Figs. 5(b,d)), respectively. Parts of interconnected vessel networks in the choroid (dark regions, black arrows) are discernible in Figs. 5(b,d). While DPV is able to capture the inner retinal vessels (Fig. 3(c) and Figs. 4(b)), multiple scattering degrades DPV contrast for SL vessels visualization (Figs. 5(b,d)).
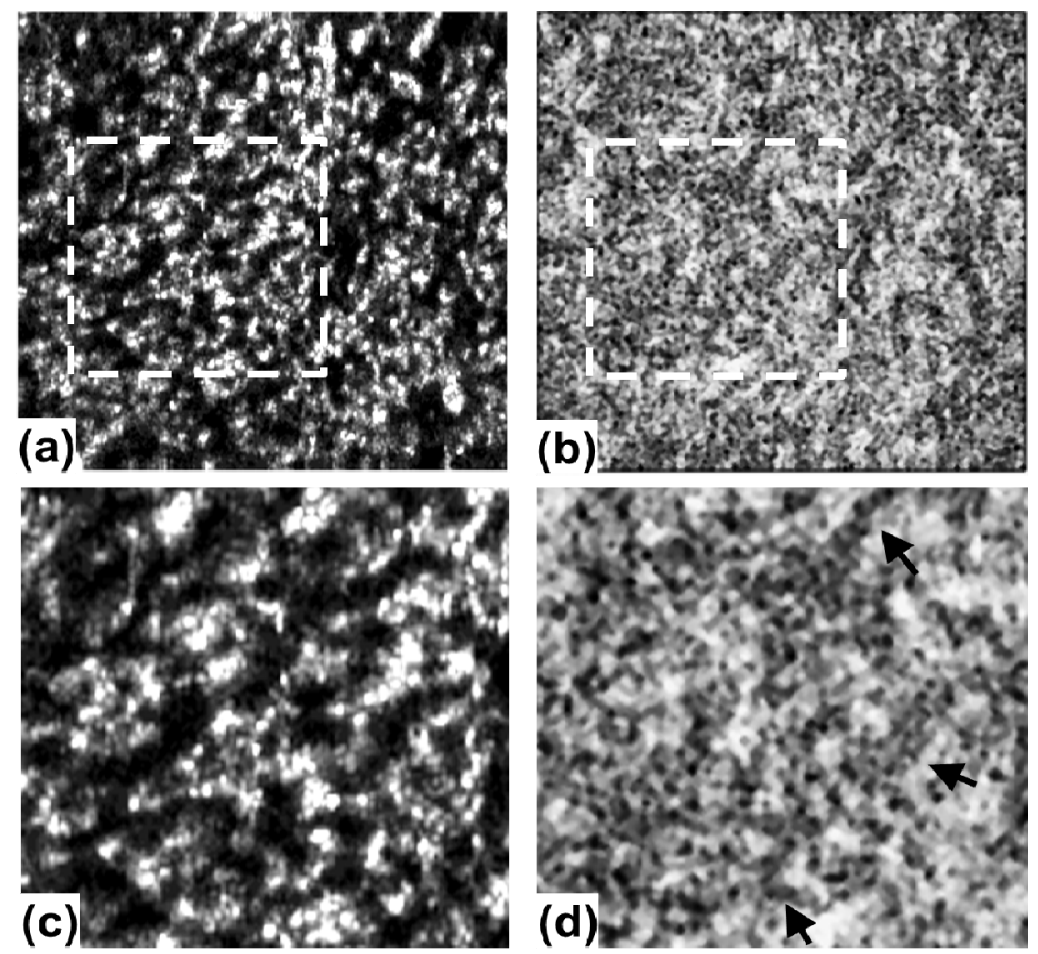

Fig. 5. Foveal depth-integrated en face views over $4 \mathrm{~mm}^{2}$ FOV acquired in 4 seconds. (a) Linear intensity, (b) inverted DPV en face images of the Sattler's layer in the choroid. Close-up views of the signified regions with dashed lines (c) in Fig. 5(a) and (d) in Fig. 5(b). 


\section{CONCLUSION}

In summary, we have demonstrated the use of a high speed SS-OCT system (50 kHz line rate) at the 1- $\mu \mathrm{m}$ wavelength region to provide enhanced penetration depth for human retinal and choroidal vasculature visualization. Our results show the potential application of depth-resolved DPV method for replacing invasive FA and ICGA in the future. While the DPV method enables us to visualize and detect small vessels and capillaries in the inner retina, the blood vessels in the inner choroid are obscured in the DPV image due to multiple scattering. It is also possible that the long time separation between B-scans causes multiple phase wrapping and Doppler signal randomization and degrades phase contrast for blood vessel visualization in highly dense vascular regions such as the inner choroid. This demonstrates the need for tailoring the optimal time separation between B-scans for capturing blood vessels in the choriocapillaris and SL in different posterior regions of the retina.

\section{ACKNOWLEDGMENTS}

This work was supported in part by research from the California Institute for Regenerative Medicine (CIRM). The authors thank Carl Zeiss Meditec for providing the patient interface part of their Stratus OCT system.

\section{REFERENCES}

1. V. Patel, S. Rassam, R. Newsom, J. Wiek, and E. Kohner, "Retinal blood flow in diabetic retinopathy." BMJ. 305(6855), 678-683 (1992).

2. E. Friedman, "A hemodynamic model of the pathogenesis of age-related macular degeneration," Am. J. Ophthalmol. 124, 677-682 (1997).

3. J. Flammer, S. Orgul, V. P. Costa, N. Orzalesi, G. K. Krieglstein, L. M. Serra, J.-P. Renard, and E. Stefansson, “The impact of ocular blood flow in glaucoma," Prog. Retin. Eye Res. 21, 359-393 (2002).

4. S. S. Hayreh, Anterior Ischemic Optic Neuropathy, (Springer-Verlag, 1975).

5. J. D. Gass, Stereoscopic atlas of macular diseases, 4th ed. (Mosby, 1997).

6. W. Drexler and J. G. Fujimoto, Optical Coherence Tomography: Technology and Applications, Biological and Medical Physics, Biomedical Engineering," (Springer, 2008).

7. M. Wojtkowski, V. Srinivasan, J. G. Fujimoto, T. Ko, J. S. Schuman, A. Kowalczyk, and J. S. Duker, "Threedimensional retinal imaging with high-speed ultrahigh-resolution optical coherence tomography," Ophthalmology 112(10), 1734-1746 (2005).

8. S. Makita, Y. Hong, M. Yamanari, T. Yatagai, and Y. Yasuno, "Optical coherence angiography," Opt. Express 14(17), 7821-7840 (2006).

9. T. Schmoll, C. Kolbitsch, and R. A. Leitgeb, "Ultra-high-speed volumetric tomography of human retinal blood flow," Opt. Express 17, 4166 (2009).

10. J. Fingler, R. J. Zawadzki, J. S. Werner, D. Schwartz, and S. E. Fraser, "Volumetric microvascular imaging of human retina using optical coherence tomography with a novel motion contrast technique," Opt. Express 17(24), 22190-22200 (2009).

11. A. Szkulmowska, M. Szkulmowski, D. Szlag, A. Kowalczyk, and M. Wojtkowski, "Three-dimensional quantitative imaging of retinal and choroidal blood flow velocity using joint Spectral and Time domain Optical Coherence Tomography,” Opt. Express 17(13), 10584-10598 (2009).

12. R. K. Wang, L. An, P. Francis, and D. J. Wilson, "Depth-resolved imaging of capillary networks in retina and choroid using ultrahigh sensitive optical microangiography," Opt. Lett. 35, 1467-1469 (2010).

13. A. Unterhuber, B. Považay, B. Hermann, A. Chavez-Pirson, and W. Drexler, "In vivo retinal optical coherence tomography at $1040 \mathrm{~nm}$ - enhanced penetration into the choroid," Opt. Express 13, 3252 (2005).

14. R. K. Wang and L. An, "Multifunctional imaging of human retina and choroid with 1050-nm spectral domain optical coherence tomography at 92-kHz line scan rate," J. Biomed. Opt. 16(5), 050503 (2011).

15. Shuichi Makita, Franck Jaillon, Masahiro Yamanari, Masahiro Miura, and Yoshiaki Yasuno, "Comprehensive in vivo micro-vascular imaging of the human eye by dual-beam-scan Doppler optical coherence angiography," Opt. Express 19, 1271-1283 (2011).

16. B. J. Vakoc, R. M. Lanning, J. A. Tyrrell, T. P. Padera, L. A. Bartlett, T. Stylianopoulos, L. L. Munn, G. J. Tearney, D. Fukumura, R. K. Jain, and B. E. Bouma, "Three-dimensional microscopy of the tumor microenvironment in vivo using optical frequency domain imaging,” Nat. Med. 15(10), 1219-1223 (2009). 
17. B. Vakoc, S. H. Yun, J. F. de Boer, G. J. Tearney, and B. E. Bouma, "Phase-resolved optical frequency domain imaging," Opt. Express 13, 5483-5493 (2005).

18. E. C. Lee, J. F. de Boer, M. Mujat, H. Lim, and S. H. Yun, "In vivo optical frequency domain imaging of human retina and choroids," Opt. Express 14, 4403 (2006).

19. M. C. Pierce, B. H. Park, B. Cense, and J. F. de Boer, "Simultaneous intensity, birefringence, and flow measurements with high-speed fiber-based optical coherence tomography," Opt. Lett. 27, 1534-1536 (2002).

20. Y. Yasuno, V. D. Madjarova, S. Makita, M. Akiba, A. Morosawa, C. Chong, T. Sakai, K.-P. Chan, M. Itoh, and T. Yatagai, "Three-dimensional and high-speed swept-source optical coherence tomography for in vivo investigation of human anterior eye segments," Opt. Express 13(26), 10652-10664 (2005).

21. B. Cense, N. Nassif, T. Chen, M. Pierce, S. H Yun, B. Park, B. Bouma, G. Tearney, and J. de Boer, "Ultrahighresolution high-speed retinal imaging using spectral-domain optical coherence tomography," Opt. Express 12, 24352447 (2004). 